Kragujevac Journal of Mathematics

Volume 44(1) (2020), Pages 113-125.

\title{
TWO-SIDED LIMIT SHADOWING PROPERTY ON ITERATED FUNCTION SYSTEMS
}

\author{
M. MOHTASHAMIPOUR ${ }^{1}$ AND A. ZAMANI BAHABADI ${ }^{1 *}$
}

\begin{abstract}
In this article, we introduce the two-sided limit shadowing property on an iterated function system $(I F S)$ and attain some results such as totally transitivity, and shadowing property. Also, by means of the strong shadowing property, we achieve topologically mixing for this IFS. Then, we study the strong two-sided limit shadowing property and obtain the topologically mixing property, immediately. Moreover, we find a criterion to obtain the two-sided limit shadowing property.
\end{abstract}

\section{Introduction AND Definitions}

To find real trajectories close to approximate trajectories, usually, the shadowing property and its various cases are used. What we want to study on iterated function systems, the systems with several generators, is two-sided limit shadowing property. Some mathematicians, like Oprocha, Carvalho and Kwietniak worked on the systems with just one generator, ordinary dynamical systems, that have this property and obtained remarkable results. For example, in [4], authors showed that systems having two-sided limit shadowing property are transitive and have the shadowing property. The relationship between two-sided limit shadowing property and another kinds of shadowing was studied in [3] and [6].

Let us mention some notations and necessary definitions on ordinary dynamical systems and iterated function systems. One can see these definitions in [2], [5], [8], and [10] for dynamical systems with one generator.

Let $(X, d)$ be a compact metric space and $f: X \rightarrow X$ be a homeomorphism. Assume that $\varepsilon$ and $\delta$ are positive integer numbers. A sequence $\left\{x_{i}\right\}_{i \in \mathbb{Z}}$ is said to be a

Key words and phrases. Iterated function system, two-sided limit shadowing property, totally transitive, topologically mixing, skew product.

2010 Mathematics Subject Classification. Primary: 37B05. Secondary: 54H20.

DOI 10.46793/KgJMat2001.113M

Received: August 29, 2017.

Accepted: February 25, 2018. 
$\delta$-pseudo trajectory, if $d\left(f\left(x_{i}\right), x_{i+1}\right)<\delta$, for all $i \in \mathbb{Z}$. This sequence is $\varepsilon$-shadowed whenever there exists $x \in X$ such that $d\left(f^{i}(x), x_{i}\right)<\varepsilon$, for all $i \in \mathbb{Z}$. We say that $f$ has the shadowing property if for every $\varepsilon>0$ there is $\delta>0$ such that every $\delta$-pseudo trajectory is $\varepsilon$-shadowed by some point of $X$.

A sequence $\left\{x_{i}\right\}_{i \in \mathbb{Z}}$ is a limit pseudo trajectory, provided that $d\left(f\left(x_{i}\right), x_{i+1}\right) \rightarrow 0$ as $i \rightarrow+\infty$, and it is limit shadowed if there is $x \in X$ such that $d\left(f^{i}(x), x_{i}\right) \rightarrow 0$ as $i \rightarrow$ $+\infty$. Also, a sequence $\left\{x_{i}\right\}_{i \in \mathbb{Z}}$ is a negative limit pseudo trajectory if $d\left(f\left(x_{i}\right), x_{i+1}\right) \rightarrow 0$ as $i \rightarrow-\infty$, and it is negative limit shadowed whenever there exists $x \in X$ such that $d\left(f^{i}(x), x_{i}\right) \rightarrow 0$ as $i \rightarrow-\infty$.

If $d\left(f\left(x_{i}\right), x_{i+1}\right) \rightarrow 0$ as $|i| \rightarrow \infty$, then $\left\{x_{i}\right\}_{i \in \mathbb{Z}}$ is called a two-sided limit pseudo trajectory. The sequence $\left\{x_{i}\right\}_{i \in \mathbb{Z}}$ is said to be two-sided limit shadowed if $d\left(f^{i}(x), x_{i}\right) \rightarrow 0$ as $|i| \rightarrow \infty$, for some $x \in X$. Principally, $f$ has the two-sided limit shadowing property while every two-sided limit pseudo trajectory is two-sided limit shadowed. Analogous definitions can be presented for limit shadowing and negative limit shadowing properties.

There is a weaker case, namely, the two-sided limit shadowing property with a gap (see [4]). A sequence $\left\{x_{i}\right\}_{i \in \mathbb{Z}}$ is two-sided limit shadowed with gap $K$ if $d\left(f^{i}(x), x_{i}\right) \rightarrow 0$ as $i \rightarrow-\infty$ and $d\left(f^{i+K}(x), x_{i}\right) \rightarrow 0$ as $i \rightarrow+\infty$, for some $x$ in $X . f$ is said to have the two-sided limit shadowing property with gap $N$ if every two-sided limit pseudo trajectory is two-sided limit shadowed with gap $K$ for $K \in \mathbb{Z}$ and $|K| \leq N$. Generally, $f$ has the two-sided limit shadowing property with a gap if there exists such $N$.

A homeomorphism $f$ is transitive whenever for every two nonempty open subsets $U$ and $V$, there is a non-negative integer $n$ such that $f^{n}(U) \cap V \neq \emptyset$. Also it is topologically mixing if for every two nonempty open subset $U$ and $V$ there is $m \in \mathbb{N}$ such that for all $n \geq m, f^{n}(U) \cap V \neq \emptyset$.

The following theorems were proved by Carvalho and Kwietniak in [4].

Theorem 1.1. If a homeomorphism $f$ of a compact metric space $X$ has the twosided limit shadowing property with a gap, then it is transitive and has the shadowing property.

Theorem 1.2. If a homeomorphism of a compact metric space has the two-sided limit shadowing property then it is topologically mixing.

They also borrowed expansivity and specification properties as tools to obtain the two-sided limit shadowing property in [3]. For definitions of expansivity and specification properties, see [3] and [7].

Theorem 1.3. Every expansive homeomorphism $f: X \rightarrow X$ with the shadowing and specification properties has the two-sided limit shadowing property.

Now, we extend some of these definitions to iterated function systems.

Let $F=\left\{f_{1}, f_{2}, \ldots, f_{k}\right\}$ be a finite sequence of homeomorphisms on compact metric space $X$. 
An iterated function system is the semigroup action generated by $F$, and denoted by $\operatorname{IFS}(F)$. The elements of $F$ are called generators of $I F S(F)$.

Let $\omega=\left(\ldots, \omega_{-1}, \omega_{0}, \omega_{1}, \ldots\right) \in\{1, \ldots, k\}^{\mathbb{Z}}$. We set $f_{\omega}^{0}=i d$, and for all $n>0$, $f_{\omega}^{n}=f_{\omega_{n-1}} o f_{\omega_{n-2}} o \ldots o f_{\omega_{0}}$ and $f_{\omega}^{-n}=f_{\omega_{-n}}^{-1} o \ldots o f_{\omega_{-1}}^{-1}$. Consider $\sigma:\{1, \cdots, k\}^{\mathbb{Z}} \rightarrow$ $\{1, \cdots, k\}^{\mathbb{Z}}, \sigma\left(\cdots, \omega_{-1}, \omega_{0}^{*}, \omega_{1}, \omega_{2}, \cdots\right)=\left(\cdots, \omega_{-1}, \omega_{0}, \omega_{1}^{*}, \omega_{2}, \cdots\right)$, be the shift map. The map

$$
\theta:\{1, \cdots, k\}^{\mathbb{Z}} \times X \rightarrow\{1, \cdots, k\}^{\mathbb{Z}} \times X, \quad \theta(\omega, x)=\left(\sigma \omega, f_{\omega_{0}}(x)\right),
$$

is called the skew product of $I F S(F)$, which $F=\left\{f_{1}, \cdots, f_{k}\right\}$.

Assume that $\varepsilon>0$ and $\delta>0$ are given. A sequence $\left\{x_{i}\right\}_{i \in \mathbb{Z}}$ is called a $\delta$-pseudo trajectory for $\operatorname{IF} S(F)$ if there exists $\omega \in\{1, \ldots, k\}^{\mathbb{Z}}$ such that

$$
d\left(f_{\omega_{i}}\left(x_{i}\right), x_{i+1}\right)<\delta,
$$

for every $i \in \mathbb{Z}$. A $\delta$-pseudo trajectory is said to be $\varepsilon$-shadowed whenever there are $y \in X$ and $\varphi \in\{1, \ldots, k\}^{\mathbb{Z}}$ such that

$$
d\left(f_{\varphi}^{i}(y), x_{i}\right)<\varepsilon,
$$

for every $i \in \mathbb{Z}$. We say that $I F S(F)$ has the shadowing property, if for every $\varepsilon>0$, there is $\delta>0$ such that every $\delta$-pseudo trajectory for $\operatorname{IFS}(F)$ is $\varepsilon$-shadowed and it has the strong shadowing property whenever $\varphi$, in the definition of the shadowing property, is equal to $\omega$, in definition of $\delta$-pseudo trajectory for $\operatorname{IFS}(F)$.

Remark 1.1. If $I F S(F)$ has the strong shadowing property, then every generator has the shadowing property but its converse is not true (see Example 1.5 in [11]).

In continuation of the previous definitions, we define limit, negative limit and two-sided limit shadowing properties on iterated function systems.

A sequence $\left\{x_{i}\right\}_{i \in \mathbb{Z}}$ is called a (negative) limit pseudo trajectory for $\operatorname{IF} S(F)$ if there exists $\omega \in\{1, \ldots, k\}^{\mathbb{Z}}$ such that

$$
d\left(f_{\omega_{i}}\left(x_{i}\right), x_{i+1}\right) \rightarrow 0,
$$

as $(i \rightarrow-\infty) i \rightarrow+\infty$. This sequence is said to be (negative) limit-shadowed if there are $y \in X$ and $\varphi \in\{1, \ldots, k\}^{\mathbb{Z}}$ such that

$$
d\left(f_{\varphi}^{i}(y), x_{i}\right) \rightarrow 0
$$

as $(i \rightarrow-\infty) i \rightarrow+\infty$. We say that $\operatorname{IFS}(F)$ has the (negative) limit shadowing property whenever every (negative) limit pseudo trajectory for $\operatorname{IFS}(F)$ is (negative) limit shadowed by some point of $X$.

A sequence $\left\{x_{i}\right\}_{i \in \mathbb{Z}}$ is called two-sided limit pseudo trajectory for $\operatorname{IFS}(F)$ if there is $\omega \in\{1, \ldots, k\}^{\mathbb{Z}}$ such that

$$
d\left(f_{\omega_{i}}\left(x_{i}\right), x_{i+1}\right) \rightarrow 0
$$


as $|i| \rightarrow \infty$. This pseudo trajectory is said to be two-sided limit shadowed while there are $y \in X$ and $\varphi \in\{1, \ldots, k\}^{\mathbb{Z}}$ such that

$$
d\left(f_{\varphi}^{i}(y), x_{i}\right) \rightarrow 0
$$

as $|i| \rightarrow \infty$. Also it is strong two-sided limit shadowed whenever $\varphi=\omega$. We say that IFS $(F)$ has the (strong) two-sided limit shadowing property whenever every two-sided limit pseudo trajectory is (strong) two-sided limit shadowed.

Remark 1.2. If an iterated function system has the strong two-sided limit shadowing property, then one can see that every its generator has the two-sided limit shadowing property.

An iterated function system is called chain transitive while for all $\delta>0$, and every $(x, y) \in X \times X$, there exist $n \in \mathbb{N}$ and a finite $\delta$-pseudo trajectory $\left\{r_{i}\right\}_{i=0}^{n}$ such that $r_{0}=x$ and $r_{n}=y$. We say that $\operatorname{IFS}(F)$ is transitive if for every two nonempty open subsets $U$ and $V$, there are $\omega \in\{1, \ldots, k\}^{\mathbb{Z}}$ and a positive integer $n$ such that $f_{\omega}^{n}(U) \cap V \neq \emptyset$. Let $\{1, \ldots, k\}^{n}$ be the set of all words of length $\mathrm{n}$. We say that $\operatorname{IF} S(F)$ is totally transitive whenever $\operatorname{IFS}\left(F^{n}\right)$ is transitive, for all $n \in \mathbb{N}$, where $F^{n}:=\left\{f_{\omega}^{n} \mid \omega \in\{1, \ldots, k\}^{n}\right\}$.

$\operatorname{IFS}(F)$ is called topologically mixing if for every two nonempty open subsets $U$ and $V$ in $X$, there is $m \in \mathbb{N}$ such that for all $n \geq m$ there exists $\omega^{n} \in\{1, \ldots, k\}^{\mathbb{Z}}$ such that $f_{\omega^{n}}^{n}(U) \cap V \neq \emptyset$. We say that $I F S(F)$ is uniformly contracting, whenever

$$
\sup _{i \in\{1, \ldots, k\}} \sup _{y \neq x} \frac{d\left(f_{i}(x), f_{i}(y)\right)}{d(x, y)}
$$

exists and is smaller than 1.

In the next section, we show that the iterated function systems equipped by the two-sided limit shadowing property are totally transitive and if these IFS's have the strong shadowing property then they also are topollogically mixing. Moreover, we find a relation between two-sided limit shadowing and shadowing properties as in the following.

Theorem A. If $\operatorname{IFS}(F)$ has the two-sided limit shadowing property, then it is totally transitive and has the shadowing property.

Theorem B. Let $I F S(F)$ has the two-sided limit shadowing and the strong shadowing properties. Then it is topologically mixing.

Moreover, we study the strong two-sided limit shadowing property on iterated function systems and obtain the topologically mixing property, immediately.

Theorem C. If $\operatorname{IFS}(F)$ has the strong two-sided limit shadowing property, then it is topologically mixing.

Also, we find a relation between iterated function system and its skew product, whenever, they have the two-sided limit shadowing property.

Theorem D. IFS $(F)$ has the strong two-sided limit shadowing property if and only if its corresponding skew product has the two-sided limit shadowing property. 
After that, in Section 3, we introduce a criterion to obtain the two-sided limit shadowing property by the following theorem.

Theorem E. Every uniformly contracting iterated function system with one to one continuous generators has the strong two-side limit shadowing property.

\section{Transitivity and Mixing Property}

Along to this section, we assume that $X$ is a compact metric space and $F=$ $\left\{f_{1}, f_{2}, \ldots, f_{k}\right\}$ is a finite sequence of homeomorphisms on $X$.

Proposition 2.1. Let IFS $(F)$ has the two-sided limit shadowing property and $F^{-1}:=$ $\left\{f_{1}^{-1}, \ldots, f_{k}^{-1}\right\}$. Then $\operatorname{IF} S(F)$ and IF $S\left(F^{-1}\right)$ have the limit shadowing property.

Proof. Let $\left\{x_{i}\right\}_{i \in \mathbb{Z}}$ be a limit pseudo trajectory for $\operatorname{IFS}(F)$ and let $\left\{z_{i}\right\}_{i \in \mathbb{Z}}$ be a limit pseudo trajectory for $I F S\left(F^{-1}\right)$. There exist $\omega, t \in\{1, \ldots k\}^{\mathbb{Z}}$ such that $d\left(f_{\omega_{i}}\left(x_{i}\right), x_{i+1}\right) \rightarrow 0$ as $i \rightarrow+\infty$ and $d\left(f_{t_{i}}^{-1}\left(z_{i}\right), z_{i+1}\right) \rightarrow 0$ as $i \rightarrow+\infty$.

Since $f_{i}, i=1, \ldots, k$, is a homeomorphism, we have $d\left(z_{i}, f_{t_{i}}\left(z_{i+1}\right)\right) \rightarrow 0$ as $i \rightarrow+\infty$. Set $z_{i+1}:=x_{-i}$, for all $i>0$. It is easily seen that $\left\{x_{i}\right\}_{i \in \mathbb{Z}}$ is a two-sided limit pseudo trajectory for $\operatorname{IF} S(F)$ with $s= \begin{cases}\omega_{i}, & i \geq 0, \\ t_{-i}, & i<0 .\end{cases}$

$\operatorname{IFS}(F)$ has the two-sided limit shadowing property, so there are $y \in X$ and $\varphi \in\{1, \ldots, k\}^{\mathbb{Z}}$ such that

$$
d\left(f_{\varphi}^{i}(y), x_{i}\right) \rightarrow 0 \text { as } i \rightarrow-\infty
$$

and

$$
d\left(f_{\varphi}^{i}(y), x_{i}\right) \rightarrow 0 \text { as } i \rightarrow+\infty .
$$

(2.1) implies that $I F S\left(F^{-1}\right)$ has the limit shadowing property and by $(2.2) \operatorname{IFS}(F)$ has the limit shadowing property.

In the following, we prove the chain transitivity of iterated function systems equipped to the two-sided limit shadowing property.

Proposition 2.2. If IFS $(F)$ has the two-sided limit shadowing property, then it is chain transitive.

Proof. Let $(x, y) \in X \times X$. Denote the $\omega$-limit set of $x$ for $f_{1}$ and $\alpha$-limit set of $y$ for $f_{1}$ by $\omega_{f_{1}}(x)$ and $\alpha_{f_{1}}(y)$, respectively.

Assume $z \in \omega_{f_{1}}(x), w \in \alpha_{f_{1}}(y)$ and $p_{n}= \begin{cases}f_{1}^{n}(z), & n<0, \\ f_{1}^{n}(w), & n \geq 0 .\end{cases}$

The sequence $\left\{p_{n}\right\}$ is a two-sided limit pseudo trajectory, so there are $p \in X$ and $\omega \in\{1, \ldots, k\}^{\mathbb{Z}}$ such that

$$
\left\{\begin{array}{l}
d\left(f_{\omega}^{i}(p), p_{i}\right) \rightarrow 0 \text { as } i \rightarrow-\infty \\
d\left(f_{\omega}^{i}(p), p_{i}\right) \rightarrow 0 \text { as } i \rightarrow+\infty
\end{array}\right.
$$


Infact,

$$
\left\{\begin{array}{l}
d\left(f_{\omega}^{i}(p), f_{1}^{i}(z)\right) \rightarrow 0 \text { as } i \rightarrow-\infty \\
d\left(f_{\omega}^{i}(p), f_{1}^{i}(w)\right) \rightarrow 0 \text { as } i \rightarrow+\infty
\end{array}\right.
$$

For $\delta>0$, there exists $M \in \mathbb{N}$ such that

$$
\left\{\begin{array}{c}
d\left(f_{\omega}^{-M}(p), f_{1}^{-M}(z)\right)<\delta / 2 \\
d\left(f_{\omega}^{M}(p), f_{1}^{M}(w)\right)<\delta / 2
\end{array}\right.
$$

Since $f_{1}^{-M}(z) \in \omega_{f_{1}}(x)$ and $f_{1}^{M}(w) \in \alpha_{f_{1}}(y)$, there are $M_{1}>0$ and $M_{2}>0$ such that

$$
\left\{\begin{array}{l}
d\left(f_{1}^{M_{1}}(x), f_{1}^{-M}(z)\right)<\delta / 2 \\
d\left(f_{1}^{-M_{2}}(y), f_{1}^{M}(w)\right)<\delta / 2
\end{array}\right.
$$

So,

$$
\left\{\begin{array}{l}
d\left(f_{\omega}^{-M}(p), f_{1}^{M_{1}}(x)\right)<\delta \\
d\left(f_{\omega}^{M}(p), f_{1}^{-M_{2}}(y)\right)<\delta
\end{array}\right.
$$

Hence, we have the chain $x, f_{1}(x), \ldots, f_{1}^{M_{1}-1}(x), f_{\omega}^{-M}(p), f_{\omega}^{-M+1}(p), \ldots, f_{\omega}^{M-1}(p)$, $f_{1}^{-M_{2}}(y), f_{1}^{-M_{2}+1}(y), \ldots, y$. It shows chain transitivity of $\operatorname{IFS}(F)$.

Now, we are ready to approximate pseudo trajectories by real trajectories.

Proposition 2.3. If IFS $(F)$ is chain transitive and has the limit shadowing property, then it has the shadowing property.

Proof. Consider $\operatorname{IFS}(F)$ does not have the shadowing property. Therefore, there exists $\varepsilon>0$ such that for every $n>0$ there is $\omega^{n} \in\{1, \ldots, k\}^{\mathbb{Z}}$ and there exists a finite $\frac{1}{n}$-pseudo trajectory $A_{n}^{\omega^{n}}$ that it cannot be $\varepsilon$-shadowed by any points of $X$. By assumption, $\operatorname{IFS}(F)$ is chain transitive, so for all $n>0$ there exist $\gamma^{n} \in$ $\{1, \ldots, k\}^{\mathbb{Z}}$ and a $\frac{1}{n}$-pseudo trajectory $B_{n}^{\gamma^{n}}$ from the end member of $A_{n}^{\omega^{n}}$ to the first member of $A_{n+1}^{\omega^{n+1}}$ and hence a finite $\frac{1}{n}$-pseudo trajectory $A_{n}^{\omega^{n}} B_{n}^{\gamma^{n}} A_{n+1}^{\omega^{n+1}}$. The sequence $\left\{y_{i}\right\}=A_{1}^{\omega^{1}} B_{1}^{\gamma^{1}} A_{2}^{\omega^{2}} B_{2}^{\gamma^{2}} \ldots$ is an infinite limit pseudo trajectory. $\operatorname{IF} S(F)$ has the limit shadowing property, so the sequence $\left\{y_{i}\right\}$ is limit shadowed by a point $y \in X$ and $\omega \in\{1, \ldots, k\}^{\mathbb{Z}}$; that is, we have $d\left(f_{\omega}^{n}(y), y_{n}\right) \rightarrow 0$ as $n \rightarrow \infty$. Hence, for every $\varepsilon>0$, there is $N \in \mathbb{N}$ such that $d\left(f_{\omega}^{n}(y), y_{n}\right)<\varepsilon$, for every $n \geq N$. This means that there exists a finite pseudo trajectory $A_{k}^{\omega^{k}}$ which is $\varepsilon$-shadowed by some point in X. It is a contradiction.

At present, we obtain the transitivity by the previous results.

Proposition 2.4. Let IFS $(F)$ has the shadowing property and let be chain transitive. Then it is transitive. 
Proof. Let $U$ and $V$ be two nonempty open subsets of $X, x \in U, y \in V$ and $\varepsilon>0$. By the shadowing property, there is $\delta>0$ such that every $\delta$-pseudo trajectory, specially the $\delta$-pseudo trajectory from $x$ to $y$, is $\varepsilon$-shadowed by a point $z \in X$. Let $x=z_{0}, z_{1}, \ldots, z_{n}=y$ be a $\delta$-pseudo trajectory for $I F S(F)$ from $x$ to $y$. So $d\left(f_{\varphi}^{i}(z), z_{i}\right)<\varepsilon$, for all $i$, some $z \in X$ and some $\varphi \in\{1, \ldots, k\}^{\mathbb{Z}}$. If we choose $\varepsilon$ small enough such that $B(x, \varepsilon) \subset U$, then $f_{\varphi}^{n}(U) \cap V \neq \emptyset$.

Proposition 2.5. If IFS $(F)$ has the two-sided limit shadowing property, then IFS $\left(F^{n}\right)$ also has the two-sided limit shadowing property, for all $n \in \mathbb{Z} \backslash\{0\}$.

Proof. Let $n \in \mathbb{Z} \backslash\{0\}$ and $\left\{x_{i}\right\}_{i \in \mathbb{Z}}$ be a two-sided limit pseudo trajectory for $\operatorname{IFS}\left(F^{n}\right)$. There is a sequence $\omega=\left\{\omega^{i}\right\}_{i \in \mathbb{Z}}, \omega^{i} \in\{1, \ldots, k\}^{n}$, such that

$$
d\left(f_{\omega^{i}}^{n}\left(x_{i}\right), x_{i+1}\right) \rightarrow 0,
$$

as $|i| \rightarrow \infty$. It is easily seen that the sequence

$$
y_{m}= \begin{cases}x_{i}, & \text { if there is } i \in \mathbb{Z} \text { such that } m=i n, \\ f_{\omega^{i}}^{m-i n}\left(x_{i}\right), & \text { if there is } i \in \mathbb{Z} \text { such that } i n<m<(i+1) n,\end{cases}
$$

is a two-sided limit pseudo trajectory for $I F S(F)$. The right elements of this sequence are

$$
\begin{aligned}
& x_{0}, f_{\omega^{0}}^{1}\left(x_{0}\right), f_{\omega^{0}}^{2}\left(x_{0}\right), \ldots, f_{\omega^{0}}^{n-1}\left(x_{0}\right), x_{1}, f_{\omega^{1}}^{1}\left(x_{1}\right), \ldots, f_{\omega^{1}}^{n-1}\left(x_{1}\right), \ldots, x_{k}, \\
& f_{\omega^{k}}^{1}\left(x_{k}\right), \ldots, f_{\omega^{k}}^{n-1}\left(x_{k}\right), \ldots
\end{aligned}
$$

We can write the rest of this sequence, similarly. $I F S(F)$ has the two-sided limit shadowing property so the sequence $\left\{y_{m}\right\}$ is two-sided limit shadowed by a point $y \in X$ and $\gamma \in \mathbb{Z}$ such that

$$
d\left(f_{\gamma}^{m}(y), y_{m}\right) \rightarrow 0
$$

as $|i| \rightarrow \infty$, that it implies $d\left(f_{\gamma}^{\text {in }}(y), x_{i}\right) \rightarrow 0$ as $|i| \rightarrow \infty$.

Proof of Theorem A. With regard to the Propositions 2.1, 2.2, 2.3 and 2.4, if IF $S(F)$ has the two-sided limit shadowing property, then it is transitive and has the shadowing property. Then the Proposition 2.5 conclude the proof of theorem A.

Proposition 2.6. If IF $S(F)$ is transitive and has the strong shadowing property, then for every nonempty open subset $U$ there exist a closed subset $B \subset U, m \in \mathbb{N} \cup\{0\}$ and $\varphi \in\{1, \cdots, k\}^{\mathbb{Z}}$ such that $f_{\varphi}^{m}(B)=B$.

Proof. Assume $U$ is a nonempty open subset of $X$. Choose $\varepsilon>0$ such that for some $u \in U, B(u, 3 \varepsilon) \subset U$. By shadowing property for every $\varepsilon>0$ there is $\delta, 0<\delta<\varepsilon$, such that every $\delta$-pseudo trajectory is $\varepsilon$-shadowed. Transitivity of $I F S(F)$ follows that there exist $n>0, x \in B(u, \varepsilon)$ and $\omega \in\{1, \ldots, k\}^{\mathbb{Z}}$ such that $d\left(f_{\omega}^{n}(x), x\right)<\delta$. The sequence $\left\{z_{m}=f_{\omega}^{m}(\bmod n)(x)\right\}$ is a $\delta$-pseudo trajectory.

So there is $z \in B(x, \varepsilon) \subset B(u, 2 \varepsilon)$ such that $d\left(f_{\varphi}^{m n}(z), x\right)<\varepsilon$ for all $m \geq 0$, when $\varphi=\left(\ldots, \omega_{0}, \omega_{1}, \ldots, \omega_{n-1}, \omega_{0}, \ldots, \omega_{n-1}, \ldots\right) \in\{1, \ldots, k\}^{\mathbb{Z}}$. 
Indeed, $\left\{z_{m}\right\}_{m \in \mathbb{Z}}$ is the following sequence

$$
\left\{\ldots, x, f_{\omega}^{1}(x), f_{\omega}^{2}(x), \ldots, f_{\omega}^{n-1}(x), x, f_{\omega}^{1}(x), f_{\omega}^{2}(x), \ldots, f_{\omega}^{n-1}(x), \ldots\right\} .
$$

The set of all limits of subsequents of $\left\{f_{\varphi}^{m n}(z): m \geq 0\right\}$ is the subset of closure of $B(u, 2 \varepsilon)$. We denote this set by $C$. Therefore, $C \subset U$. Since, here and for this $\varphi$, $f_{\varphi}^{m n}=\left(f_{\varphi}^{n}\right)^{m}$ and $\omega$-limit set of $f_{\varphi}^{n}$ is $f_{\varphi}^{n}$-invariant, $f_{\varphi}^{n}(C)=C$.

Now, it is prepared some qualifications to obtain topologically mixing IFS's.

Proposition 2.7. If IFS $(F)$ has the strong shadowing property and is totally transitive, then it is topologically mixing.

Proof. Let $U$ and $V$ are two nonempty open subsets of $X$. Choose $\varepsilon>0$ so that $U_{1}=B(u, 2 \varepsilon) \subset U$ and $V_{1}=B(v, 2 \varepsilon) \subset V$, for some $u \in U$ and $v \in V$.

By the shadowing property, there is $\delta<\varepsilon / 2$ such that every $\delta$-pseudo trajectory is $\varepsilon$-shadowed.

$\operatorname{IFS}(F)$ is transitive so for $U_{2}=B(u, \delta / 2)$ and $V_{1}$ there exist $n \in \mathbb{N} \cup\{0\}$ and $w \in\{1, \ldots, k\}^{\mathbb{Z}}$ such that $f_{w}^{n}\left(U_{2}\right) \cap V_{1} \neq \varnothing$. By Proposition 2.6, there exist a closed subset $B \subset U_{2} \cap\left(f_{w}^{n}\right)^{-1} V_{1}, m \in \mathbb{N} \cup\{0\}$ and $\varphi \in\{1, \ldots, k\}^{\mathbb{Z}}$ that $f_{\varphi}^{m}(B)=B$. Also, $f_{w}^{n}\left(f_{\varphi}^{m}\right)^{j}\left(U_{2}\right) \cap V_{1} \neq \varnothing$, for all $j \in \mathbb{N} \cup\{0\}$. So, for every $j \in \mathbb{N} \cup\{0\}$, there is $r^{j} \in\{1, \ldots, k\}^{\mathbb{Z}}$ such that $f_{r^{j}}^{n+m j}\left(U_{2}\right) \cap V_{1} \neq \varnothing$.

Set $G:=F^{m}$. Transitivity of $I F S(G)$ implies that for given $\gamma \in\{1, \ldots, k\}^{\mathbb{Z}}$ and integer $s \geq 0$, there is $j_{s} \geq 0$ and $\xi^{s} \in\{1, \ldots, k\}^{\mathbb{Z}}$ such that $f_{x i^{s}}^{m j_{s}}\left(f_{\gamma}^{s}\left(U_{2}\right)\right) \cap\left(f_{w}^{n}\right)^{-1} V_{1} \neq$ $\varnothing$. Hence, there exists $\eta^{s} \in\{1, \ldots, k\}^{\mathbb{Z}}$ such that $f_{\eta^{s}}^{n+m j_{s}+s}\left(U_{2}\right) \cap V_{1} \neq \varnothing$.

Set $J_{s}:=\min \left\{j_{s} \mid f_{\eta^{s}}^{n+m j_{s}+s}\left(U_{2}\right) \cap V_{1} \neq \varnothing\right.$, for some $\left.\eta^{s} \in\{1, \ldots, k\}^{\mathbb{Z}}\right\}$ and $M:=$ $\max \left\{n+m J_{s} \mid 0 \leq s \leq m-1\right\}$.

We claim that for all $l \geq M$ there is $\theta^{l} \in\{1, \ldots, k\}^{\mathbb{Z}}$ such that $f_{\theta^{l}}^{l}\left(U_{2}\right) \cap V_{1} \neq \varnothing$. For this aim, consider $l \geq M$. So there exist $j \geq 0$ and $0 \leq s \leq m-1$ such that $l=n+m j+s$. Since $l \geq M, j \geq J_{s}$. Therefore, $l-m p=n+m J_{s}+s$ for some $p \geq 0$. The sequence

$$
y_{l, t}= \begin{cases}f_{\varphi}^{t}(\bmod m)(b), & 0 \leq t \leq m p-1, \\ f_{\eta^{s}}^{t-m p}\left(y^{s}\right), & m p \leq t,\end{cases}
$$

where $b \in B$ and $y^{s} \in U_{2} \cap\left(f_{\eta^{s}}^{n+m J_{s}+s}\right)^{-1} V_{1} \neq \varnothing$, is a $\delta$-pseudo trajectory. So it is $\varepsilon$-shadowed with a point $y^{l} \in X$ and $\theta^{l} \in\{1, \ldots, k\}^{\mathbb{Z}}$. In fact, $d\left(f_{\theta^{l}}^{t}\left(y^{l}\right), y_{l, t}\right)<\varepsilon$ for all $t \geq 0$.

When $t=0$, we have $d\left(y^{l}, b\right)<\varepsilon$ so $y^{l} \in U$.

If $t=l$, then $d\left(f_{\theta^{l}}^{l}\left(y^{l}\right), f_{\eta^{s}}^{n+m J_{s}+s}\left(y^{s}\right)\right)<\varepsilon$ and since $f_{\eta^{s}}^{n+m J_{s}+s}\left(y^{s}\right) \in V_{1}, f_{\theta^{l}}^{l}\left(U_{2}\right) \cap$ $V_{1} \neq \varnothing$. Therefore $\operatorname{IFS}(F)$ is topologically mixing.

We are now ready to prove Theorem B.

Proof of Theorem B. By Theorem A, IFS $(F)$ is totally transitive and because it has the strong shadowing property, Proposition 2.7 implies it is topologically mixing. 
Here, we want to study about the strong two-sided limit shadowing property on iterated function systems as a stronger property than the two-sided limit shadowing property.

Similar to proof of the Proposition 2.1, we have the following proposition.

Proposition 2.8. If IFS $(F)$ has the strong two-sided limit shadowing property, Then it has the strong limit shadowing property.

We can use a similar proof of the Proposition 2.3 to obtain the following result.

Proposition 2.9. If IFS $(F)$ is chain transitive and has the strong limit shadowing property, then it has the strong shadowing property.

Proposition 2.10. If IFS $(F)$ has the strong two-sided limit shadowing property, Then it is totally transitive and has the strong shadowing property.

Proof. Since an iterated function system with the strong two-sided limit shadowing property has the two-sided limit shadowing property, by Theorem A, it is totally transitive and obviousely chain transitive; and, by Propositions 2.8 and 2.9, it has the strong shadowing property.

Now, by using the strong two-sided limit shadowing, we can prove Theorem C.

Proof of Theorem C. By Proposition 2.10, IFS $(F)$ has the strong shadowing property. Theorem B completes the proof.

In the following, we prove Theorem $\mathrm{D}$ to show a relation between iterated function systems having the strong two-sided limit shadowing property and their skew product. Proof of Theorem D. First, assume that $\operatorname{IFS}(F)$ has the strong two-sided limit shadowing property and $\left\{\left(\omega^{i}, x^{i}\right)\right\}_{i \in \mathbb{Z}}$ is a two-sided limit pseudo trajectory for $\theta$ where

$$
\theta:\{1, \ldots, k\}^{\mathbb{Z}} \times X \rightarrow\{1, \ldots, k\}^{\mathbb{Z}} \times X, \quad \theta(\omega, x)=\left(\sigma \omega, f_{\omega_{0}}(x)\right),
$$

is the skew product of $\operatorname{IFS}(F)$.

Consider metrics $D, d_{1}$ and $d$ on $\{1, \ldots, k\}^{\mathbb{Z}} \times X,\{1, \ldots, k\}^{\mathbb{Z}}$ and $X$ respectively and

We have

$$
D((w, x),(\varphi, y))=\max \left\{d_{1}(w, \varphi), d(x, y)\right\}
$$

$$
D\left(\theta\left(\omega^{i}, x^{i}\right),\left(\omega^{i+1}, x^{i+1}\right)\right) \rightarrow 0 \text { as }|i| \rightarrow \infty,
$$

that is, $D\left(\left(\sigma \omega^{i}, f_{w_{0}^{i}}\left(x^{i}\right)\right),\left(\omega^{i+1}, x^{i+1}\right)\right) \rightarrow 0$ as $|i| \rightarrow \infty$. It is equivalent to

$$
\begin{aligned}
& d_{1}\left(\sigma \omega^{i}, \omega^{i+1}\right) \rightarrow 0 \text { as }|i| \rightarrow \infty, \\
& d\left(f_{\omega_{0}^{i}}\left(x^{i}\right), x^{i+1}\right) \rightarrow 0 \text { as }|i| \rightarrow \infty .
\end{aligned}
$$

The expression (2.3) says that the sequence $\left\{\omega^{i}\right\}_{i \in \mathbb{Z}}$ is a two-sided limit pseudo trajectory. This sequence is two-sided limit shadowed by $\varphi=\left(\ldots, \omega_{0}^{-1}, \omega_{0}^{* 0}, \omega_{0}^{1}, \ldots\right)$, (see Theorem 5.1 in [4]). Infact, we have

$$
d_{1}\left(\sigma^{i}(\varphi), \omega^{i}\right) \rightarrow 0 \text { as }|i| \rightarrow \infty
$$


Equation (2.4) implies that $\left\{x^{i}\right\}$ is a two-sided limit pseudo trajectory for $I F S(F)$. Since $I F S(F)$ has the strong two-sided limit shadowing property, there exists a point $x \in X$ such that that

$$
d\left(f_{\varphi}^{i}(x), x^{i}\right) \rightarrow 0 \text { as }|i| \rightarrow \infty .
$$

The statements (2.5) and (2.6) imply that

$$
D\left(\theta^{i}(\varphi, x),\left(\omega^{i}, x^{i}\right)\right) \rightarrow 0 \text { as }|i| \rightarrow \infty .
$$

Conversly, assume $\left\{x^{i}\right\}$ is a two-sided limit pseudo trajectory for $\operatorname{IFS}(F)$. There is $\omega \in\{1, \ldots, k\}^{\mathbb{Z}}$ such that

$$
d\left(f_{\omega_{i}}\left(x^{i}\right), x^{i+1}\right) \rightarrow 0 \text { as }|i| \rightarrow \infty
$$

It is obvious that the sequence $\left\{\varphi^{i}=\sigma^{i} \omega\right\}_{i \in \mathbb{Z}}$ is a two-sided limit pseudo trajectory and it is two-sided limit shadowed by $\omega$. Therefore, $\left\{\left(\varphi^{i}, x^{i}\right)\right\}_{i \in \mathbb{Z}}$ is a two-sided limit pseudo trajectory for $\theta$ and is two-sided limit shadowed by some $x \in X$ and $\gamma=\left(\ldots, \varphi_{0}^{-1}, \varphi_{0}^{* 0}, \varphi_{0}^{1}, \ldots\right)=\omega$. So we have $d\left(f_{\omega}^{i}(x), x^{i}\right) \rightarrow 0$ as $|i| \rightarrow \infty$, and it means $\operatorname{IFS}(F)$ has the strong two-sided limit shadowing property.

As an application of the Theorem $\mathrm{D}$, we present the following example to show that the inverse of Theorem $\mathrm{B}$ and Theorem $\mathrm{C}$ are not true.

Example 2.1. Suppose that $X=[0,1]$ and $f_{0}: X \rightarrow X, f_{0}(x)=0$ and $f_{1}: X \rightarrow X$, $f_{1}(x)=1-|1-2 x|$. Let $\theta$ be the skew product of $\operatorname{IFS}\left(\left\{f_{0}, f_{1}\right\}\right)$. IFS $\left(\left\{f_{0}, f_{1}\right\}\right)$ is topologically mixing but $\theta$ is not topologically mixing (see Example 1 in [9]). Theorem B in [4] implies that $\theta$ does not have the two-sided limit shadowing property. By Theorem D, IFS $\left(\left\{f_{0}, f_{1}\right\}\right)$ does not have the strong two-sided limit shadowing property. Therefore, we have an iterated function system that is topologically mixing but does not have the strong two-sided limit shadowing property. This implies the inverse of Theorem $\mathrm{C}$ is not true. Moreover, $\operatorname{IFS}\left(\left\{f_{0}, f_{1}\right\}\right)$ dose not have the strong shadowing property (see Example 1.3 in [11]). So, the inverse of Theorem B does not hold.

\section{A CRIterion}

Assume that $X$ is a compact metric space and $F=\left\{f_{1}, f_{2}, \ldots, f_{k}\right\}$ is a finite sequence of one to one continuous functions from $\mathrm{X}$ to itself.

Proof of Theorem E. Suppose that $I F S(F)$ is uniformly contracting,

$$
\beta=\sup _{i \in\{1, \ldots, k\}} \sup _{x \neq y} \frac{d\left(f_{i}(x), f_{i}(y)\right)}{d(x, y)}<1,
$$

and the sequence $\left\{x_{i}\right\}_{i \in \mathbb{Z}}$ is a two-sided limit pseudo trajectory for $\operatorname{IFS}(F)$. There is $w \in\{1, \ldots, k\}^{\mathbb{Z}}$ such that $d\left(f_{w_{n}}\left(x_{n}\right), x_{n+1}\right) \rightarrow 0$ as $|n| \rightarrow \infty$.

Choose the sequence $\left\{y_{i}\right\}_{i \in \mathbb{Z}}$ so that $y_{0}=x_{0}$ and $y_{i+1}=f_{w_{i}}\left(y_{i}\right), i \in \mathbb{Z}$. In Theorem 3.2 in [1], it is proved that every uniformly contracting $I F S$ has the limit shadowing 
property, and also $d\left(f_{w}^{n}\left(y_{0}\right), x_{n}\right) \rightarrow 0$ as $n \rightarrow \infty$. Here, we show that $d\left(f_{w}^{n}\left(y_{0}\right), x_{n}\right) \rightarrow 0$ as $n \rightarrow-\infty$.

Set $\theta_{i}:=d\left(f_{w_{i}}\left(x_{i}\right), x_{i+1}\right), i \in \mathbb{Z}$. We have

$$
\begin{aligned}
d\left(x_{-1}, y_{-1}\right) & \leq d\left(f_{w_{-2}}\left(y_{-2}\right), f_{w_{-2}}\left(x_{-2}\right)\right)+d\left(f_{w_{-2}}\left(x_{-2}\right), x_{-1}\right) \\
& \leq \beta d\left(y_{-2}, x_{-2}\right)+\theta_{-2}
\end{aligned}
$$

and

$$
\begin{aligned}
d\left(x_{-2}, y_{-2}\right) & \leq d\left(f_{w_{-3}}\left(y_{-3}\right), f_{w_{-3}}\left(x_{-3}\right)\right)+d\left(f_{w_{-3}}\left(x_{-3}\right), x_{-2}\right) \\
& \leq \beta d\left(x_{-3}, y_{-3}\right)+\theta_{-3} .
\end{aligned}
$$

By induction,

$$
d\left(x_{-n}, y_{-n}\right) \leq \beta d\left(x_{-(n+1)}, y_{-(n+1)}\right)+\theta_{-(n+1)} .
$$

Set $\gamma_{n}:=\theta_{-n}$, for $n \geq 0 . \gamma_{n} \rightarrow 0$ as $n \rightarrow \infty$. So, for given $\varepsilon>0$, there is $T \in \mathbb{N}$ such that for all $n \geq T$, we have $\gamma_{n}<\frac{\varepsilon(1-\beta)}{2}$. Assume that $a_{n}:=d\left(x_{-n}, y_{-n}\right)$, for all $n>0$. Since $X$ is compact, the sequence $\left\{a_{n}\right\}$ has a convergent subsequence $\left\{a_{n_{k}}\right\}_{k \geq 0}$. This fact and $\beta<1$ imply that for all $n_{k} \geq T$, the following inequalities hold:

$$
\begin{aligned}
a_{n_{k}} & \leq \beta^{n_{k+1}-n_{k}} a_{n_{k+1}}+\beta^{n_{k+1}-n_{k}-1} \gamma_{n_{k+1}}+\cdots+\gamma_{n_{k}+1} \\
& \leq \beta^{n_{k+1}-n_{k}} a_{n_{k+1}}+\frac{\varepsilon}{2}(1-\beta)\left(1+\cdots+\beta^{n_{k+1}-n_{k}-1}\right) \\
& \leq \beta a_{n_{k+1}}+\frac{\varepsilon}{2}\left(1-\beta^{n_{k+1}-n_{k}-1}\right) \\
& \leq \beta a_{n_{k+1}}+\varepsilon .
\end{aligned}
$$

As $k \rightarrow \infty$, we have $a \leq \beta a+\frac{\varepsilon}{2}$. $\varepsilon$ is arbitrary so $a(1-\beta) \leq 0$ and therefore $a=0$.

Now, we claim that every subsequence of the sequence $\left\{a_{n}\right\}$ is convergent to zero. Consider a subsequence $\left\{a_{n_{t}}\right\}_{t \geq 0}$ such that $a_{n_{t}} \nrightarrow 0$ as $t \rightarrow 0$. This subsequence has a subsequence $\left\{a_{n_{t_{l}}}\right\}_{l \geq 0}$ such that $a_{n_{t_{l}}}>\varepsilon$ for all $l(*)$.

$X$ is compact, so $\left\{a_{n_{t_{l}}}\right\}_{l \geq 0}$ has a convergent subsequence $\left\{a_{n_{t_{l}}}\right\}_{k \geq 0}$. Similar to before the claim, we have $a_{n_{t_{l_{k}}}} \rightarrow 0$ as $k \rightarrow \infty$. This contradicts $(*)$. Hence, $a_{n} \rightarrow 0$ as $n \rightarrow \infty$. Namely, $\lim _{n \rightarrow \infty} d\left(x_{-n}, y_{-n}\right)=0$. It means that $\lim _{n \rightarrow-\infty} d\left(f_{w}^{n}\left(y_{0}\right), x_{n}\right)=0$. Therefore, $d\left(f_{w}^{n}\left(y_{0}\right), x_{n}\right) \rightarrow 0$ as $|n| \rightarrow \infty$.

Example 3.1. Let $d_{1}:\{0,1\}^{\mathbb{Z}} \times\{0,1\}^{\mathbb{Z}} \rightarrow \mathbb{R}$

$$
d_{1}(w, \varphi)=\sup _{i \in \mathbb{Z}} \frac{\delta\left(w_{i}, \varphi_{i}\right)}{2^{|i|}},
$$

be a metric for $\{0,1\}^{\mathbb{Z}}$, where

$$
w=\left(\ldots, w_{-1}, w_{0}^{*}, w_{1}, \ldots\right), \quad \varphi=\left(\ldots, \varphi_{-1}, \varphi_{0}^{*}, \varphi_{1}, \ldots\right), \quad \delta\left(w_{i}, \varphi_{i}\right)= \begin{cases}1, & w_{i} \neq \varphi_{i} \\ 0, & w_{i}=\varphi_{i}\end{cases}
$$

Assume that $f_{0}:\{0,1\}^{\mathbb{Z}} \rightarrow\{0,1\}^{\mathbb{Z}}$,

$$
f_{0}\left(\ldots, w_{-1}, w_{0}^{*}, w_{1}, \ldots\right)=\left(\ldots, \varphi_{-1}, \varphi_{0}^{*}, \varphi_{1}, \ldots\right),
$$


such that

and $f_{1}:\{0,1\}^{\mathbb{Z}} \rightarrow\{0,1\}^{\mathbb{Z}}$

$$
\varphi_{i}= \begin{cases}w_{i-1}, & i \geq 1 \\ 1, & i=0,-1 \\ w_{i+1}, & i \leq-1\end{cases}
$$

$$
f_{1}\left(\ldots, w_{-1}, w_{0}^{*}, w_{1} \ldots\right)=\left(\ldots, \gamma_{-1}, \gamma_{0}^{*}, \gamma_{1}, \ldots\right)
$$

such that

$$
\gamma_{i}= \begin{cases}w_{i-1}, & i \geq 1 \\ 0, & i=0,-1, \\ w_{i+1}, & i<1\end{cases}
$$

One can see $\operatorname{IFS}\left(f_{0}, f_{1}\right)$ is uniformly contracting and by Theorem A, it has the strong two-sided limit shadowing property. Moreover, Proposition 3.1 implies that the skew product of $\operatorname{IFS}\left(f_{0}, f_{1}\right)$ has the two-sided limit shadowing property.

Let $Y$ be a compact metric space, $G=\left\{g_{1}, \ldots, g_{k}\right\}$ and let $g_{i}: Y \rightarrow Y$ be a homeomorphism, for $i=1, \ldots, k$.

We say that $I F S(F)$ and $I F S(G)$ are conjugate if there exists a homeomorphism $h: X \rightarrow Y$ such that $h \circ f_{i}=g_{i} \circ h$, for $i=1, \ldots, k$. It can be proved easily that conjugacy preserves the two-sided limit shadowing property. So if $\operatorname{IFS}(F)$ has the twosided limit shadowing property and it is conjugate to $\operatorname{IF} S(G)$, then $\operatorname{IFS}(G)$ has the two-sided limit shadowing property, too. The proof of this subject is straightforward, so we eliminate it.

Acknowledgements. The authors would like to thank the respectful referee for his/her comments on the manuscript. Also we would like to thank F.H. Ghane for her useful conversations.

\section{REFERENCES}

[1] S. A. Ahmadi and M. Fatehi Nia, Various shadowing properties for parameterized iterated function system, University Polytehnica of Bucharest, The Scientific Bulletin, Series A 80(1) (2018), 145-154.

[2] N. Aoki and K. Hiraide, Topological Theory of Dynamical Systems, Recent Advances, Mathematical Library 52, North-Holland Publishing Co. Amsterdam, 1994.

[3] B. Carvalho, Hyperbolicity, transitivity and two-sided limit shadowing property, Proc. Amer. Math. Soc. 143(2) (2015), 657-666.

[4] B. Carvalho and D. Kwietniak, On homeomorphisms with two-sided limit shadowing property, J. Math. Anal. Appl. 420(1) (2014), 801-813.

[5] H. Furstenberg, Disjointness in ergodic theory, minimal sets, and a problem in Diophantine approximation, Mathematical Systems Theory 1 (1967), 1-49.

[6] D. Kwietniak and D. Oprocha, A note on average shadowing property for expansive maps, Topology Appl. 159(1) (2012), 19-27.

[7] R. Mane, Expansive homeomorphisms and topological dimension, Trans. Amer. Math. Soc. 252 (1979), 313-319. 
[8] K. Palmer, Shadowing in Dynamical Systems: Theory and Applications, Mathematics and its Applications 501, Kluwer Academic Publishers, Dordrecht, 2000.

[9] X. Wu, L. Wang and J. Liang, The chain properties and average shadowing property of iterated function systems, Qual. Theory Dyn. Syst. 17(1) (2018), 219-227.

[10] S. Yu. Pilyugin, Shadowing in Dynamical Systems, Lecture Notes in Mathematics 1706, Springer, Berlin, 1999.

[11] A. Zamani Bahabadi, Shadowing and average shadowing properties for iterated function systems, Georgian Math. J. 22(2) (2015), 179-184.

${ }^{1}$ Department of Pure Mathematics, FERDOWSI UNIVERSITY OF MASHHAD, MASHHAD, IRAN

Email address: ma_mo149@mail.um.ac.ir

Email address: zamany@um.ac.ir

*Corresponding Author 